\title{
EXTENDING DISCRETE-VALUED FUNCTIONS
}

\author{
JOHN KULESZA, RONNIE LEVY, AND PETER NYIKOS
}

\begin{abstract}
In this paper, we show that for a separable metric space $X$, every continuous function from a subset $S$ of $X$ into a finite discrete space extends to a continuous function on $X$ if and only if every continuous function from $S$ into any discrete space extends to a continuous function on $X$. We also show that if there is no inner model having a measurable cardinal, then there is a metric space $X$ with a subspace $S$ such that every 2 -valued continuous function from $S$ extends to a continuous function on all of $X$, but not every discrete-valued continuous function on $S$ extends to such a map on $X$. Furthermore, if Martin's Axiom is assumed, such a space can be constructed so that not even $\omega$-valued functions on $S$ need extend. This last result uses a version of the Isbell-Mrowka space $\Psi$ having a $C^{*}$-embedded infinite discrete subset. On the other hand, assuming the Product Measure Extension Axiom, no such $\Psi$ exists.
\end{abstract}

\section{INTRODUCTION}

Suppose that $X$ is a metric space and $S$ is a subset of $X$. Then the following statements are equivalent: (i) Every continuous function $f: S \rightarrow \mathbb{R}$ extends to a continuous function $F: X \rightarrow \mathbb{R}$, and (ii) Every continuous function $f: S \rightarrow \mathbb{R}$ having a compact image extends to a continuous function $F: X \rightarrow \mathbb{R}$. (Each statement is equivalent to $S$ being a closed subset of $X$. To see this, notice that (i) trivially implies (ii), and every closed set satisfies (i) by Tietze's Extension Theorem. Finally, if $S$ is not closed in $X$, there is a sequence $\sigma$ in $S$ converging to a point $p$ not in $X$. Then any function $f: \sigma \rightarrow\{0,1\}$ such that each fiber is infinite extends to a continuous $F: S \rightarrow[0,1]$. If $F$ is not onto, it can be composed with a continuous function from the range of $F$ onto $\{0,1\}$. In any case, we get a continuous function from $S$ onto a compact set which does not extend continuously to $p$.) In this paper we deal with the question of what happens when $\mathbb{R}$ is replaced by a discrete space. In particular, we discuss the following problem: Suppose that $X$ is a metric space and $S$ is a subset such that every continuous function $f: S \rightarrow\{0,1\}$ extends to a continuous function $F: X \rightarrow\{0,1\}$. Does it follow that every continuous function from $S$ to a discrete space extends to a continuous function defined on all of $X$ ? We show that if $X$ is separable, then the answer is "yes".

Received by the editors September 30, 1988 and, in revised form, March 14, 1989.

1980 Mathematics Subject Classification (1985 Revision). Primary 54C20, 54E35.

Key words and phrases. $\Psi$, PMEA, $\alpha$-embedding. 
However, if $X$ fails to be separable, the situation becomes clouded by settheoretic considerations. We show that if there is no inner model having a measurable cardinal, then there is a metric space and a subset such that every $\{0,1\}$-valued continuous function extends, but not every function having a discrete image extends. Furthermore, assuming something weaker than Martin's Axiom, there is a metric space and a subset such that every $\{0,1\}$-valued function extends, but not every $\omega$-valued function extends. The construction of both of these examples involves finding a first countable space which has a certain clopen reflection, and then applying a technique which gives a metric space having the same clopen reflection as that space. It is shown that assuming the Product Measure Extension Axiom, these examples cannot be constructed by this technique.

We wish to thank the referee for many helpful suggestions.

\section{Preliminaries.}

We will use standard set-theoretic and topological terminology. See [E], [GJ], $[\mathrm{K}]$, and $[\mathrm{W}]$ for notions not defined here. Cardinals (that is, initial ordinals) will be assumed to have the discrete topology. If $X$ is a topological space, $S$ is a subset of $X$, and $\alpha$ is a cardinal number, then $S$ is $\alpha$-embedded in $X$ if every continuous function $f: S \rightarrow \alpha$ extends to a continuous function $F: X \rightarrow \alpha$. Clearly, if $\alpha \leq \gamma$ and $S$ is $\gamma$-embedded in $X$, then $S$ is $\alpha$ embedded in $X$. It follows from the Urysohn Extension Theorem that every 2-embedded discrete subset of a topological space is $C^{*}$-embedded.

Suppose that $\alpha$ is an infinite cardinal. Then the hedgehog with $\alpha$ spines, denoted $H(\alpha)$, is the subspace $\left\{c e_{\lambda} \in l_{2}(\alpha): 0 \leq c \leq 1, e_{\lambda} \in \mathscr{B}\right\}$ of $l_{2}(\alpha)$, where $\mathscr{B}$ is the standard orthonormal basis for the Hilbert space $l_{2}(\alpha)$. The convex hull of $\left\{0, e_{\lambda}\right\}$ is called the $\lambda$ th spine, and $e_{\lambda}$ is called the end of the $\lambda$ th spine.

An infinite collection $\mathfrak{E}$ of infinite subsets of $\omega$ is almost disjoint if every pair of distinct elements of $\mathfrak{E}$ has finite intersection. $\mathfrak{E}$ is a maximal almost disjoint (MAD) family if $\mathfrak{E}$ is almost disjoint and is not a proper subset of any almost disjoint family of subsets of $\omega$. It is easy to see that $\mathscr{E}$ is an almost disjoint family if and only if the collection $\left\{\left(\mathrm{Cl}_{\beta \omega} E\right) \backslash E: E \in \mathscr{E}\right\}$ is a disjoint collection of clopen subsets of $\omega^{*}$, and that $\mathscr{E}$ is a MAD family if and only if $\left\{\left(\mathrm{Cl}_{\beta \omega} E\right) \backslash E: E \in \mathscr{E}\right\}$ is a disjoint collection of clopen subsets of $\omega^{*}$ whose union is dense in $\omega^{*}$. Given a MAD family $\mathscr{E}$, there is a pseudocompact space $\Psi_{\mathscr{E}}$ whose points are $\omega \cup\left\{p_{E}: E \in \mathscr{E}\right\}$ where each element of $\omega$ is isolated and a typical neighborhood of $p_{E}$ is $\left\{p_{E}\right\} \cup(E \backslash F)$ where $F$ is a finite subset of $E$. Another way of describing $\Psi_{\mathscr{E}}$ is that it is obtained from the subspace $\omega \cup\left\{\left(\mathrm{Cl}_{\beta \omega} E\right) \backslash E: E \in \mathscr{E}\right\}$ of $\beta \omega$ by collapsing each element of $\left\{\left(\mathrm{Cl}_{\beta \omega} E\right) \backslash E: E \in \mathscr{E}\right\}$ to a single point. We call any space of the form $\Psi_{\mathscr{E}}$ a version of $\Psi$. 
If $\alpha$ is a cardinal number, an $\alpha$-additive measure on a $\sigma$-algebra $\Sigma$ of subsets of a set $S$ is a nonnegative real-valued function $\mu$ defined on $\Sigma$ with the property that given any collection $\mathscr{C}$ of fewer than $\alpha$ pairwise disjoint elements of $\Sigma$, at most countably many elements, say $C_{1}, C_{2}, \ldots$, satisfy $\mu\left(C_{k}\right)>0$, and $\mu\left(\bigcup C_{k}\right)=\sum \mu\left(C_{k}\right)$. The Product Measure Extension Axiom, abbreviated PMEA, is the following statement: Given any set $X$, there is a C-additive measure $\mu$ on the power set $\mathscr{P}(X)$ of $X$ with the property that if $F$ is any finite subset of $X$, then $\mu\{A \subseteq X: F \subseteq A\}=1 / 2^{|F|}$. It is known that the consistency of the existence of a strongly compact cardinal implies the consistency of PMEA.

The organization of the paper is as follows. In $\S 2$, we prove that if $X$ is a separable metric space and $S$ is a subset of $X$, then $S$ is 2-embedded in $X$ if and only if $S$ is $\alpha$-embedded in $X$ for every cardinal $\alpha$. A consequence is that if $X$ is a separable metric space, $S$ is a subset of $X$, and $Y$ is a subset of $\mathbb{R}$, then every continuous function $f: S \rightarrow Y$ extends to a continuous function $F: X \rightarrow Y$ if and only if every bounded continuous function $f: S \rightarrow Y$ extends to a continuous function $F: X \rightarrow Y$. In $\S 3$, we discuss a process which we call hedgehogging a first countable space. The idea of hedgehogging a first countable space is to replace each point of the space with a hedgehog and topologizing the resulting set in such a way that the topology is metrizable and has the same zero-dimensional reflection as the original space. Hedgehogging certain familiar spaces gives consistent examples of metric spaces having 2-embedded subsets which are not $\alpha$-embedded for certain cardinals $\alpha$. We also construct a consistent example of a version of $\Psi$ which has a 2-embedded infinite discrete subset. Hedgehogging this version of $\Psi$ gives a consistent example of a metric space having a 2-embedded subset which is not $\omega$-embedded. In $\S 4$, we give some consequences of PMEA. In particular, we show that assuming PMEA, in many cases the zero-dimensional reflection of a space cannot have a 2-embedded subset which is not $\alpha$-embedded. It will follow that, assuming PMEA, in many cases a metric space, or more generally a first countable space, cannot have a 2 -embedded subset which is not $\alpha$-embedded. Furthermore, assuming PMEA, a pseudocompact space of small character cannot have an infinite discrete $C^{*}$ embedded subset, so, in particular, there cannot be a version of $\Psi$ having an infinite 2-embedded discrete subset.

\section{SeParable Metric SPACES}

In this section, we show that if $S$ is a subset of a separable metric space $X$, then if every 2-valued continuous function from $S$ extends to such a function from $X$, then for each cardinal $\alpha$, every $\alpha$-valued continuous function from $S$ extends to such a function from $X$. It will be helpful to use the following observation: If $\alpha$ is a cardinal number and $X$ is any topological space, then a continuous function from $X$ to $\alpha$ gives rise to a partition of $X$ into clopen subsets, one for each nonempty fiber. Conversely, every partition of a space into clopen subsets induces a continuous function into a discrete space by mapping 
the elements of the partition to different points of the discrete space. Thus, for example, to say that each 2-valued continuous function from $S$ extends to a 2-valued continuous function on $X$ says exactly that every disconnection of $S$ is the trace on $S$ of a disconnection of $X$.

2.1. Proposition. Suppose that $X$ is a separable metric space and $S$ is a 2embedded subset of $X$. Then $S$ is $\omega$-embedded in $X$.

Proof. Suppose that $S$ is a 2-embedded subset of the separable metric space $X$ and $f: S \rightarrow \omega$ is a continuous function. For each $n \in \omega$, let $C_{n}=f^{\leftarrow}(n)$. Then $\left\{C_{n}: n \in \omega\right\}$ is a partition of $S$ into clopen subsets of $S$. We must show that there is a partition $\left\{A_{n}: n \in \omega\right\}$ of $X$ into clopen subsets of $X$ such that for each $n \in \omega, A_{n} \cap S=C_{n}$. Since every 2-valued continuous function on $S$ extends to a 2-valued continuous function on $X$, for each $n \in \omega$, there exists a clopen subset $W_{n}$ of $X$ such that $W_{n} \cap S=C_{n}$. Let $U_{0}=W_{0}$ and for $n>0$, let $U_{n}=W_{n} \backslash \bigcup\left\{U_{k}: k<n\right\}$. Then the sets $U_{n}$ are pairwise disjoint clopen subsets of $X$ such that $U_{n} \cap S=C_{n}$ for all $n$. Let $L$ be the boundary in $X$ of the set $\bigcup\left\{U_{k}: k \in \omega\right\}$, that is, $L=\{x \in X$ : Every neighborhood of $x$ in $X$ intersects $U_{n}$ for infinitely many $\left.n \in \omega\right\}$. For $x \in L$ and $n=1,2, \ldots$, let $B_{n}^{x}=\left\{k \in \omega\right.$ : Every clopen neighborhood of $C_{k}$ in $X$ intersects $\left.S_{1 / n}(x)\right\}$, where $S_{\varepsilon}(x)$ is the open $\varepsilon$-sphere of $x$ in $X$. Notice that for $x \in L$ and $n$ a positive integer, $B_{n}^{x} \supseteq B_{n+1}^{x}$. We now show that given an $x \in L$, there is an $m$ such that $B_{m}^{x}$ finite. For if not, we can find $k_{1}, k_{2}, \ldots$ such that $k_{i} \neq k_{j}$ for $i \neq j$ and $k_{i} \in B_{i}^{x}$. Define $g: S \rightarrow 2$ by $g(z)=0$ if $z \in C_{k_{2 i}}$ for some $i$, and $g(z)=1$ otherwise. Let $G: X \rightarrow 2$ be a continuous extension of $g$. Then either $G(x)=0$ or $G(x)=1$. Since the argument is similar in both cases, assume that $G(x)=1$. By continuity of $G$, there exists a positive integer $n$ such that $G$ is identically 0 on $S_{1 / n}(x)$. By the definitions of $B_{m}^{x}$ and the $k_{i}$ 's, there exists an integer $i$ such that every clopen neighborhood of $C_{k_{2 i+1}}$ intersects $S_{1 / n}(x)$, a contradiction of the fact that $G$ is identically 0 on $S_{1 / n}(x)$ and identically 1 on $C_{k_{2 i+1}}$. Therefore, there exists an integer $m$ such that $B_{m}^{x}$ is finite, say $B_{m}^{x} \subseteq\left\{0,1, \ldots, m_{0}^{x}\right\}$. Let $V_{x}=S_{1 / m}(x) \backslash \bigcup\left\{U_{i}: 0 \leq i \leq m_{0}^{x}\right\}$. Then $V_{x}$ is a neighborhood of $x$ in $X$ such that there exist clopen neighborhoods $W_{n}^{x}$ of $C_{n}$ in $X$ with $V_{x} \cap \bigcup_{n \in \omega}\left(W_{n}^{x}\right)=\varnothing$. By intersecting the sets $W_{n}^{x}$ with $U_{n}$, we may assume that $W_{n}^{x} \subset U_{n}$ for all $x \in L$ and for all $n \in \omega$. The open cover $\left\{V_{x}: x \in L\right\}$ has a countable subcover, say $\left\{V_{x_{0}}, V_{x_{1}}, \ldots\right\}$. Let $R_{k}=\bigcap\left\{W_{k}^{x_{i}}: i=1, \ldots, k\right\}$. Then $\bigcup_{k \in \omega} R_{k}$ is clopen, so $F: X \rightarrow \omega$ given by $F(x)=k$ if $x \in S_{k}, F(x)=0$ otherwise is a continuous extension of $f$.

We remark that the above proof works if $X$ is assumed to be any Lindelöf first countable space. At first glance, it might seem that hereditary Lindelöfness is required because a countable subcover is required for an open cover of the subset $L$ of $X$. However, $L$ is closed in $X$, so $L$ is Lindelöf if $X$ is. 
We also remark that a separable metric space hereditarily has countable celluarity. Therefore, no subset of a separable metric space admits a continuous map onto an uncountable discrete space. Therefore, Proposition 2.1 actually gives the result that a 2-embedded subset of a separable metric space is $\alpha$-embedded for every cardinal $\alpha$. Furthermore, it is easy to see that if a subset of any space is $\gamma$-embedded for some cardinal $\gamma$, then it is $\alpha$-embedded for every $\alpha<\gamma$.

Before giving the next result, we point out an example. Let $X$ be the Euclidean plane, let $S$ be the closure in $X$ of the graph of the function $s:(0, \infty) \rightarrow \mathbb{R}$ given by $s(x)=\frac{\sin (1 / x)}{x}$, and let $Y=S$. Then if $f: S \rightarrow Y$ is a bounded continuous function, the image of $f$ will be a subset of either the graph of $s$ or of the $y$-axis. It follows from Tietze's Extension Theorem that $f$ has an extension to a continuous function $F: X \rightarrow Y$. On the other hand, the identity function $l: S \rightarrow Y$ does not have an extension to a continuous function from $X$ to $Y$, because $S$ is not arcwise connected, whereas $X$ is. The point of the next result is that this sort of example cannot exist if $Y$ is a subset of $\mathbb{R}$.

2.2. Proposition. Suppose that $X$ is a separable metric space and $S$ is a subset of $X$. Let $Y$ be a subset of $\mathbb{R}$ such that every bounded continuous function $f: S \rightarrow Y$ extends to a continuous function $F: X \rightarrow Y$. Then every continuous function $f: S \rightarrow Y$ extends to a continuous function $F: X \rightarrow Y$.

Proof. We may obviously assume that $Y$ is an unbounded proper subset of $\mathbb{R}$. Since for any given $r \in Y$, the space $\{y-r: y \in Y\}$ is homeomorphic to $Y$, we may assume that $0 \in Y$. Suppose that $f: S \rightarrow Y$ is continuous. We may assume that $f$ is bounded below by 0 , because if any such function extends to a continuous function on $X$, then we can extend $f^{+}=0 \vee f$ to $F^{+}: X \rightarrow Y$ and $f^{-}=0 \vee(-f)$ to $F^{-}: X \rightarrow Y$. Then $F=F^{+}-F^{-}$will be a continuous extension of $f$. Under the assumption that $f$ is bounded below by 0 , we consider two cases.

Case (i). If $Y$ contains an interval of the form $(b, \infty)$ where $b>0$, then $S$ is closed in $X$, because in this case, the assumption that every bounded continuous function from $S$ to $Y$ extends to a continuous function on $X$ implies that $S$ is $C^{*}$-embedded in $X$. Let $h: Y \rightarrow[0, b+2)$ be a homeomorphism such that $h(y)=y$ for each $y \in Y \cap[0, b+1]$. Then $h^{\rightarrow}(Y) \subset Y$, so $h \circ f$ maps $S$ into a bounded subset of $Y$. By assumption, $h \circ f$ extends to a continuous function $g: X \rightarrow Y$. Then $G: X \rightarrow[0, b+2]$ given by $G=g \wedge(b+2)$ is also a continuous extension of $h \circ f$. By the normality of $X$, we can find a continuous function $k: X \rightarrow\left[\frac{b+1}{b+2}, 1\right]$ such that $k(x)=1$ for each $x$ in $G^{\leftarrow}[0, b+1]$ and $k(x)=\frac{b+1}{b+2}$ for each $x$ in $G^{\leftarrow}(\{b+2\})$. Then $k \cdot G$ maps $X$ into $[0, b+2)$ and the restriction of $k \cdot G$ to $S$ is $h \circ f$. Now let $F=h^{-1} \circ(k \cdot G)$. Then $F$ maps $X$ to $Y, F$ is continuous, and $F$ extends $f$.

Case (ii). Suppose that $Y$ contains no interval which is cofinal in $\mathbb{R}$. Then $Y$ can be written as the union of a discrete family of nonempty clopen subsets, 
$C_{n}, n \in \omega$, of $Y$, where every element of $C_{n+1}$ is larger than every element of $C_{n}$ for each $n$. Choose $y_{n} \in C_{n}$. Then $\Omega=\left\{y_{n}: n \in \omega\right\}$ is homeomorphic to $\omega$. If $g: S \rightarrow \Omega$ is continuous and assumes only finitely many values, then $g$ extends to a continuous function $G: X \rightarrow \Omega$. To see this, notice that such a $g$ extends to a continuous $g^{\prime}: X \rightarrow Y$ by assumption, so if $h: Y \rightarrow \Omega$ is given by $h(y)=y_{n}$ for $y \in C_{n}$, then we can let $G=h \circ g^{\prime}$. Therefore, by Proposition 2.1, every continuous function $g: S \rightarrow \Omega$ extends to a continuous function $G: X \rightarrow \Omega$. Translating this into terms of clopen partitions, it means that any partition of $S$ into clopen sets arises as the trace on $S$ of a partition of $X$ into clopen sets. Now let $B_{n}=f^{\leftarrow}\left(C_{n}\right)$. Then $\left\{B_{n}: n \in \omega\right\}$ is a partition of $S$ into clopen sets, so there is a partition $\left\{A_{n}: n \in \omega\right\}$ of $X$ into clopen sets so that $A_{n} \cap X=B_{n}$ for all $n$. For $n \in \omega$, let $f_{n}: S \rightarrow Y$ be defined by $f_{n}(x)=f(x)$ if $x \in B_{n}, f_{n}(x)=0$ otherwise. Then each $f_{n}$ is bounded and continuous so by assumption, each $f_{n}$ extends to a continuous function $F_{n}: X \rightarrow Y$. Now define $F: X \rightarrow Y$ by $F(x)=F_{n}(x)$ if $x \in A_{n}$. Then $F$ is a continuous extension of $f$.

\section{HEDGEHOGgING FIRST COUNTABLE SPACES}

In this section, we show how to replace the points of a space with hedgehogs in such a way that the clopen structure of the new space is the same as the clopen structure of the original space, and the new space is metrizable. More precisely, we define a metrizable topology on $X \times H$, where $H$ is a hedgehog, such that the zero-dimensional reflection of $X \times H$ is the same as the zero-dimensional reflection of $X$. (The zero-dimensional reflection of a space is obtained from a space by identifying each quasicomponent of the space to a point and giving the resulting set the topology obtained by declaring a set open if its inverse image under the identification function is clopen.)

Let $X$ be a first countable space and let $\xi=|X|$. Let $H=H(\xi)$ be the hedgehog on $\xi$ spines and label the spines of $H$ as $S_{x}, x \in X$. Denote the end of the spine $S_{x}$ as $p_{x}$. Define a topology on $X \times H$ as follows. A neighborhood of $(x, y)$ where $y \neq p_{x}$ is $\{x\} \times V$ where $V$ is a neighborhood of $y$ in $H$. A neighborhood of $\left(x, p_{x}\right)$ is $U \times V$ where $U$ is a neighborhood of $x$ and $V$ is a neighborhood of $p_{x}$. We will denote the resulting space $\mathscr{H}(X)$, and call this space the hedgehog of $X$.

We will use Frink's Metrization Theorem (see [N]): A Hausdorff space $Z$ is metrizable if and only if for each $p \in Z$, there is a countable nested open neighborhood base $\left\{U_{p}(n): n \in \omega\right\}$ at $p$ such that for each $n \in \omega$, there exists an $m \in \omega$ such that $U_{p}(m) \cap U_{q}(m) \neq \varnothing$ implies that $U_{q}(m) \subseteq U_{p}(n)$. We will be applying this theorem to $\mathscr{H}(X)$ for $X$ a first countable space.

3.1. Proposition. If $X$ is first countable, then $\mathscr{H}(X)$ is metrizable.

Proof. For each $p \in X$, let $\left\{V_{p}(n): n \in \omega\right\}$ be a nested local base at $p$ in $X$, and for each $q \in H$ and $n \in \omega$, let $S_{q}(n)$ denote $\{h \in H$ : the distance in $H$ from $h$ to $q$ is less than $\left.\frac{1}{n+1}\right\}$. Then for $(x, y) \in \mathscr{H}(X)$, define a nested local 
base $\left\{U_{(x, y)}(n): n \in \omega\right\}$ as follows:

(i) If $y \neq p_{x}, U_{(x, y)}(n)=\{x\} \times S_{y}(n)$, and

(ii) If $y=p_{x}, U_{(x, y)}=V_{x}(n) \times S_{y}(n)$.

Observe that $\{x\} \times H$ is just a copy of the metric space $H$ and for $y \neq p_{x}$, $U_{(x, y)}(n)$ is just the set of points in $\{x\} \times H$ at a distance of less than $\frac{1}{n+1}$ from $(x, y)$.

We will show that the $U$ 's satisfy the conditions of Frink's Theorem. Suppose that $(x, y) \in \mathscr{H}(X)$ and $n \in \omega$ are given. First suppose $y \notin S_{x}$. Then let $\tilde{y}$ be the point of $X$ such that $y \in S_{\widetilde{y}}$. (If $y=0$, choose $\tilde{y}$ arbitrarily.) Choose $r \geq n$ so that $V_{\tilde{y}}(r)$ does not contain $x$. Then if $U_{\left(x^{\prime}, y^{\prime}\right)}(m) \cap U_{(x, y)}(m) \neq \varnothing$, it follows that $x^{\prime}=x$ and $y^{\prime}=p_{x}$. Now choose $m$ so that $\frac{3}{m+1}<\frac{1}{r+1}$. If $U_{\left(x, y^{\prime}\right)}(m) \cap U_{(x, y)}(m) \neq \varnothing$, then the distance in $\{x\} \times H$ from a point $(a, b)$ in $U_{\left(x, y^{\prime}\right)}$ to $(x, y)$ is less than $\frac{3}{m+1}$ which is less than $\frac{1}{r+1}$. Since $U_{(x, y)}(r)$ is the set of points whose $\{x\} \times H$ distance to $(x, y)$ is less than $\frac{1}{r+1}$, it follows that $U_{\left(x, y^{\prime}\right)}(m) \subseteq U_{(x, y)}(r)$.

Now suppose $y \in S_{x}$ and $y \neq p_{x}$. This case is similar to the previous one, only this time choose $r$ so that the $H$-distance from $y$ to $p_{x}$ is greater than $\frac{2}{r+1}$. (This guarantees that if $U_{\left(x^{\prime}, y^{\prime}\right)}(r) \cap U_{(x, y)}(r) \neq \varnothing$, then $y^{\prime} \neq p_{x^{\prime}}$.) Now proceed as in the previous case. Finally, if $y=p_{x}$, choose $m$ so that $\frac{3}{m+1}<$ $\frac{1}{r+1}$. If $U_{\left(x^{\prime}, y^{\prime}\right)}(m) \cap U_{(x, y)}(m) \neq \varnothing$ and $\left(x^{\prime}, y^{\prime}\right) \neq(x, y)$, then $y^{\prime} \neq p_{x^{\prime}}$ (since, in that case $U_{\left(x^{\prime}, y^{\prime}\right)} \subseteq V_{x^{\prime}}(m) \times S_{x^{\prime}}$, but $\left.S_{x^{\prime}} \cap S_{x}=\varnothing\right)$. Thus, $U_{\left(x^{\prime}, y^{\prime}\right)}(m)=$ $\left\{x^{\prime}\right\} \times S_{y^{\prime}}(m), x^{\prime} \in V_{x}(m)$, and $S_{y}(m) \cap S_{y^{\prime}}(m) \neq \varnothing$, so $S_{y^{\prime}}(m) \subseteq S_{y}(r)$; therefore, $U_{\left(x^{\prime}, y^{\prime}\right)} \subseteq V_{x}(m) \times S_{y}(r) \subseteq U_{(x, y)}(r)$.

3.2. Lemma. If $D$ is a discrete subset of the space $X$ and $\alpha$ is a cardinal number, then $D$ is $\alpha$-embedded in $X$ if and only if $\left\{\left(x, p_{x}\right): x \in D\right\}$ is $\alpha$ embedded in $\mathscr{H}(X)$.

Proof. For each $f: D \rightarrow \alpha$ let $h_{f}:\left\{\left(x, p_{x}\right): x \in D\right\} \rightarrow \alpha$ be defined by $h_{f}\left(\left(x, p_{x}\right)\right)=f(x)$. The lemma will be proved if we show that $f$ extends to a continuous function $F: X \rightarrow \alpha$ if and only if $h_{f}$ extends to $H_{f}: \mathscr{H}(x) \rightarrow \alpha$. If $f$ extends to $F: X \rightarrow \alpha$, define $H_{f}: \mathscr{H}(X) \rightarrow \alpha$ by $H_{f}((x, y))=F(x)$.

Now suppose $H_{f}: \mathscr{H}(x) \rightarrow \alpha$ extends $h_{f}$. Observe that $H_{f}$ is constant on each $\{x\} \times H$ because $\alpha$ is discrete and $\{x\} \times H$ is connected. Thus $F(x)=H_{f}((x, y))$ where $(x, y)$ is any element of $\{x\} \times H$ is well-defined. $F$ is continuous because if $F(x)=\delta$, then $H_{f}^{\leftarrow}\{\delta\}$ contains a neighborhood $V_{x}(n) \times S_{p_{x}}(n)$ of $\left(x, p_{x}\right)$, since $H_{f}$ is continuous and thus contains $V_{x}(n) \times H$. Therefore, $F\left(V_{x}(n)\right)=\delta$.

Combining 3.1 and 3.2 allows us to find examples of metric spaces with 2-embedded subsets which are not $\alpha$-embedded by finding first countable spaces with these properties. In particular, assuming that no inner model has a measurable cardinal, Fleissner has constructed a normal Moore space which 
has a family $\left\{C_{\lambda}: \lambda \in \alpha\right\}$ of closed subsets which exhibits noncollectionwise normality [F]. The $C_{\lambda}$ 's are totally disconnected. If we cone over each $C_{\lambda}$, that is, replace each $C_{\lambda}$ by $C_{\lambda} \times[0,1]$ with $C_{\lambda} \times\{1\}$ identified to a point for each $\lambda$, we get a 2-embedded subset which for some infinite cardinal $\alpha$ is not $\alpha$-embedded, namely, the "points" of the cones. From this example, we get the following.

3.3. Example. If there is no inner model with a measurable cardinal, then there is a metric space $X$, a cardinal $\alpha$, and a subset of $X$ which is 2-embedded in $X$ but not $\alpha$-embedded in $X$.

Example 3.3 does not give a metric space which has a 2-embedded subset which is not $\omega$-embedded. To get such an example from 3.1 and 3.2, we need a first countable space having a 2-embedded subset which is not $\omega$-embedded. Recall that $\mathfrak{p}$ is the minimal cardinality of a centered family of clopen subsets of $\omega^{*}$ whose intersection has empty interior in $\omega^{*}$. We will use a hypothesis which follows from the hypothesis that $\mathfrak{p}=\mathfrak{c}$, to construct a version of $\Psi$ having this property. (Of course, the hypothesis $\mathfrak{p}=\mathfrak{c}$ follows from Martin's Axiom.) We note that since $\Psi$ is pseudocompact, it suffices to find a version of $\Psi$ having an infinite, discrete, 2-embedded subset. For the definitions of the cardinals $\mathfrak{b}$, and $\mathfrak{s}$, see $[\mathrm{vD}]$.

3.4. Proposition. Assuming $\mathfrak{b}=\mathfrak{s}=\mathfrak{c}$, there is a MAD family $\mathscr{E}$ such that every countable set of nonisolated points of $\Psi_{\mathscr{E}}$ is 2-embedded in $\Psi_{\mathscr{E}}$.

Proof. Let $\left\{A_{\alpha}: \alpha<\mathfrak{c}\right\}$ list all countable subsets of $\mathfrak{c}$ such that $A_{n} \subseteq \omega$ for all finite $n, A_{\alpha} \subseteq \alpha$ for all infinite $\alpha$, and $A_{\alpha}$ is listed $\mathfrak{c}$ times for all $\alpha$. Let $\left\{Z_{\alpha}: \alpha<\mathfrak{c}\right\}$ list the infinite subsets of $\omega$. Let $\left\{S_{n}: n \in \omega\right\}$ be an almost disjoint collection of infinite subsets of $\omega$. For each $\alpha \leq \omega$, let $D_{\alpha}$ be a subset of $\omega$ almost containing all $S_{n}$ with $n \in A_{\alpha}$ and almost disjoint from all $S_{n}$ with $n \notin A_{\alpha}$.

We will find collections $\left\{S_{\alpha}: \alpha<\mathfrak{c}\right\}$ and $\left\{D_{\alpha}: \alpha<\mathfrak{c}\right\}$ extending those above and satisfying, for all $\gamma$ such that $\omega \leq \gamma<\mathfrak{c}$ :

(i) $\left\{S_{\alpha}: \alpha<\gamma\right\}$ is an almost disjoint collection such that for $\omega \leq \alpha<\gamma$, $S_{\alpha} \subseteq Z_{\alpha}$, and $S_{\alpha}$ is nonempty if and only if $S_{\alpha}$ is infinite and $Z_{\alpha} \cap S_{\beta}$ is finite for each $\beta<\alpha$ and, for $\beta<\alpha, S_{\alpha}$ is almost contained in or is almost disjoint from $D_{\beta}$.

(ii) For each $\alpha \leq \mathfrak{c}, D_{\alpha} \subseteq \omega$ and $D_{\alpha}$ almost contains all $S_{\beta}$ with $\beta \in A_{\alpha}$ and is almost disjoint from all $S_{\beta}$ with $\beta \in \alpha \backslash A_{\alpha}$.

Proceeding inductively, suppose $\gamma<\mathfrak{c}$ and $\left\{S_{\alpha}: \alpha<\gamma\right\}$ and $\left\{D_{\alpha}: \alpha<\gamma\right\}$ are as above. If $Z_{\gamma} \cap S_{\beta}$ is infinite for some $\beta<\gamma$ then let $S_{\gamma}=\varnothing$. Otherwise, using $\mathfrak{s}=\mathfrak{c}$, there is an infinite subset $S_{\gamma}$ of $Z_{\gamma}$ such that for each $\alpha<\gamma, S_{\gamma}$ is either almost contained in or almost disjoint from $D_{\alpha}$. Using $\mathfrak{b}=\mathfrak{c}$, and Theorem 3.3 of [vD], we can choose $D_{\gamma}$ to satisfy condition (ii).

Claim (1). $\mathscr{E}=\left\{S_{\alpha}: \alpha<\mathfrak{c}\right\}$ is a MAD family. For suppose that $X$ is an infinite subset of $\omega$. Then $X=Z_{\alpha}$ for some $\alpha<\mathfrak{c}$. If $S_{\alpha}=\varnothing$, by (i) 
$\omega=\left|Z_{\alpha} \cap S_{\beta}\right|=\left|X \cap S_{\beta}\right|$ for some $\beta<\alpha$. By (i) $S_{\alpha}$ is empty or infinite; in either case, $X$ has infinite intersection with an element of $\mathscr{E}$.

Claim (2). If $N$ is a countable subset of the nonisolated points of $\Psi_{\mathscr{E}}$, then $N$ is 2-embedded in $\Psi_{\mathscr{E}}$. To prove this, notice first that $N$ can by written as $\left\{S_{\alpha}: \alpha \in H\right\}$ for some countable subset $H$ of $\mathrm{c}$. Let $\beta=\sup \{\alpha: \alpha \in H\}$. Let $f: N \rightarrow\{0,1\}$ be a function. Then $N=N_{0} \cup N_{1}$ where $N_{i}=f^{\leftarrow}\{i\}$ for $i=0,1$, and there are sets $H_{0}$ and $H_{1}$ such that $N_{i}=\left\{S_{\alpha}: \alpha \in H_{i}\right\}$ for $i=0,1$. There is $\gamma \geq \beta$ with $A_{\gamma}=H_{0}$; it follows that $H_{1} \subseteq \gamma \backslash A_{\gamma}$. Thus $D_{\gamma}$ almost contains each element of $N_{0}$ and is almost disjoint from each member of $N_{1}$. Since each $S_{\alpha}$ is almost contained in $D_{\gamma}$ or almost disjoint from it, $\mathrm{Cl} D_{\alpha}$ is a clopen subset of $\Psi_{\mathscr{E}}$. Thus, $F: \Psi_{\mathscr{E}} \rightarrow\{0,1\}$ defined by $F(x)=0$ if $x \in \mathrm{Cl} D_{\gamma}$ and $F(x)=1$ otherwise is a continuous extension of $f$.

We remark that a consequence of Urysohn's Extension Theorem is that a discrete 2-embedded subset of a topological space is $C^{*}$-embedded. Hence, Proposition 3.4 establishes the consistency of the existence of a version of $\Psi$ having an infinite, $C^{*}$-embedded discrete subset.

3.5. Corollary. Assuming $\mathfrak{b}=\mathfrak{s}=\mathfrak{c}$ there is a metric space which has a 2embedded subset which is not $\omega$-embedded.

\section{A CONSEQUENCE OF PMEA}

In $\S 3$, we saw that, given the right set theoretic assumptions, it is possible to find a metric space having a subset which is 2 -embedded but not $\omega$-embedded. The key to the construction is the construction of a pseudocompact first countable space which has an infinite 2-embedded subset. In this section, we show that assuming PMEA, no such pseudocompact space can exist. In fact, a somewhat stronger result holds.

4.1. Theorem. Assuming PMEA, a space $X$ of character less than $\mathfrak{c}$ is pseudocompact if and only if $X$ has no infinite $C^{*}$-embedded discrete subset.

Proof. $X$ is pseudocompact if and only if $X$ has no $C$-embedded copy of $\omega$. (See $[\mathrm{GJ}]$.) Hence, a space which has no $C^{*}$-embedded copy of $\omega$ is pseudocompact.

Now suppose $P=\left\{p_{i}: i \in \omega\right\}$ is a $C^{*}$-embedded discrete subset of $X$. We will find a discrete family $\left\{\mathscr{O}_{i}: i \in \omega\right\}$ of open sets with $p_{i} \in \mathscr{O}_{i}$ for all $i \in \omega$. For each $S \in \mathscr{P}(P)$, let $f_{S}: X \rightarrow[0,1]$ be such that $f_{S} \mid S=0$ and $f_{S} \mid(P \backslash S)=1$, and for each $x \in X$ choose a neighborhood $N_{S}(x)$ of $x$ such that the diameter of $f_{S}\left(N_{S}(x)\right)<1 / 4$. Let $\mu$ denote the measure guaranteed by PMEA. Using PMEA and the fact that the character of $X$ is less than $\mathfrak{c}$, we can find a neighborhood $N(x)$ of $x$ such that $\mu\left(\left\{S: N(x) \subseteq N_{S}(x)\right\}\right)$ $>15 / 16$. We claim that $\left\{N\left(p_{i}\right): i \in \omega\right\}$ is a discrete family. Suppose $N(x) \cap N\left(p_{i}\right) \neq \varnothing$. Then $\mu\left(\left\{S: f_{S}(N(x))\right.\right.$ is within $1 / 2$ of $\left.f_{S}\left(p_{i}\right)\right\} \geq$ $7 / 8$. For distinct $p_{i}, p_{j}, \mu\left(\left\{S: f_{S}\left(p_{i}\right) \neq f_{S}\left(p_{j}\right)\right\}\right)=1 / 2$. Thus $\mu\left(\left\{S: f_{S}(N(x))\right.\right.$ misses the $1 / 2$-neighborhood of $\left.\left.f_{S}\left(p_{j}\right)\right\}\right) \geq 3 / 8$. So $N(x) \cap N\left(p_{j}\right)=\varnothing$. Thus $\left\{N\left(p_{i}\right): i \in \omega\right\}$ is discrete. 
4.2. Corollary. Assuming PMEA, no first countable pseudocompact space has an infinite 2-embedded discrete subset.

Of course, it does not follow from 4.2 that assuming PMEA every 2-embedded subset of a metric space is $\omega$-embedded, but only that an example cannot be constructed using the method of $\S 3$. We do not know if there is a ZFC example of a metric space having a 2-embedded subset which is not $\alpha$-embedded for some $\alpha$.

We point out that the full strength of PMEA was not used to prove 4.1, but rather the statement that the product measure on $2^{\omega}$ extends to a c-additive measure defined on the collection of all subsets of $2^{\omega}$. This statement is equivalent to the statement that Lebesgue measure on the real line extends to a cadditive measure $\mu$ with the property that every subset of $\mathbb{R}$ is $\mu$-measurable.

We close with a question. We have seen that if there is no inner model having a measurable cardinal, then there is a metric space having a 2-embedded subset which is not $\alpha$-embedded. Is there a ZFC example of a metric space having a 2-embedded subset which is not $\alpha$-embedded? Specifically, is there a ZFC example of a metric space having a 2-embedded subset which is not $\omega$-embedded?

\section{REFERENCES}

[vD] E. K. van Douwen, The integers and topology, Handbook of Set Theoretic Topology, NorthHolland, Amsterdam, New York, and Oxford, 1984, pp. 113-167.

[E] R. Engelking, General topology, Polish Scientific Publishers, Warsaw, 1977.

[F] W. G. Fleissner, Normal Moore spaces and large cardinals, Handbook of Set Theoretic Topology, North-Holland, Amsterdam, New York, and Oxford, 1984, pp. 733-760.

[GJ] L. Gillman and M. Jerison, Rings of continuous functions, Van Nostrand, Princeton, N.J., 1960.

[K] K. Kunen, Set theory: An introduction to independence proofs, North-Holland, Amsterdam, 1979.

[N] K. Nagami, Dimension theory, Academic Press, New York, 1970.

[W] S. Willard, General topology, Addision-Wesley, 1970.

Department of Mathematics, George Mason University, 4400 University Drive, FairFAX, VIRGINIA 22030 (Current address of John Kulesza and Ronnie Levy)

Department of Mathematics, University of South Carolina, Columbia, South CaroLINA 19208 (Current address of Peter Nyikos) 